\title{
The two classes of low-energy spectra in finite carbon nanotubes
}

\author{
Magdalena Marganska, ${ }^{1, *}$ Piotr Chudzinski, ${ }^{1,2}$ and Milena Grifoni ${ }^{1}$ \\ ${ }^{1}$ Institute of Theoretical Physics, Regensburg University, 93053 Regensburg, Germany \\ ${ }^{2}$ Institute for Theoretical Physics, Leuvenlaan 4, 3584 CE Utrecht, The Netherlands
}

(Received 24 December 2014; revised manuscript received 7 July 2015; published 21 August 2015)

\begin{abstract}
We study the spectra of finite-length carbon nanotubes (CNTs) of arbitrary chirality. They divide into two classes, which arise because of different rotational symmetries of the low-energy eigenstates. In one of them (the zigzag class), the spinless spectrum is doubly degenerate and the two states can be assigned to different values of the valley degree of freedom. In the other (armchair class), the valley degeneracy is removed and the eigenstates are combinations of both valley states. Recent experimental observation of the valley mixing in ultraclean CNT quantum dots is consistent with our theory for armchair-class CNTs.
\end{abstract}

DOI: 10.1103/PhysRevB.92.075433

PACS number(s): 73.63.Fg, 71.70.Ej, 73.22.-f

\section{INTRODUCTION}

Research on carbon nanotubes (CNTs) as active elements in nanoelectronic devices is steadily increasing. While the electronic properties of infinitely long CNTs have been studied in detail [1-3], not much is known of the spectrum of CNTs of finite length. This knowledge, however, is crucial both from the fundamental point of view as well as for applications, e.g., in CNT-based quantum-dot devices [4-7]. In this latter case, the CNT's bound-state spectrum crucially influences the current-voltage characteristics [5,7]. At present, quantization conditions yielding the proper spectrum of bound states are only known for the so-called armchair [8-10] and zigzag $[11,12]$ CNTs. These achiral CNTs have a high degree of symmetry and well-defined edges, for which boundary conditions are easily imposed. The spectrum of finite-length chiral CNTs - the most common realization of a CNT [13] still poses a challenge. In this work, we first identify the symmetries of finite chiral CNTs and, using this knowledge, evaluate their eigenfunctions and spectrum. Our major finding is that all finite-length CNTs belong to one of two classes, i.e., an armchair (A) class or the zigzag (Z) class [14], with crucially different eigenstates and spectra. The major difference between the two classes involves the so-called valley degree of freedom, possessed by electrons in graphenelike systems. The two valleys $K$ and $K^{\prime}$ correspond to the two Dirac points of the hexagonal lattice. The spectrum of a (spinless) (Z)-class CNT consists of doubly degenerate levels, which can be assigned to pure valley states. In the (A)-class CNTs, the valley degeneracy is removed, and the eigenstates are $K / K^{\prime}$ mixtures.

The possibility of breaking the valley degeneracy through different kinds of hard wall boundaries was pointed out in Ref. [15], and through asymmetric velocities of left and right movers in Ref. [16]. In the present work, we reveal and explore the deep relation between a CNT's chirality and the nature of the spectrum of a finite nanotube.

In Sec. II, we introduce our model Hamiltonian and discuss the symmetries and spectra of infinite CNTs. The zigzag and armchair classes are also defined here. In Sec. III, we construct the spectrum of finite-length CNTs, providing the momentum quantization conditions for a minimal boundary [17]. We

\footnotetext{
*magdalena.marganska@physik.uni-r.de
}

compare those to the results of a numerical diagonalization of our Hamiltonian, analyzing the dependence of the level splitting found in the (A)-class CNTs on their length and the position of the level above the charge neutrality point (CNP). In Sec. IV, we include in our discussion the electron spin and show the results of numerical calculations of transmission through both CNT classes in parallel magnetic field, yielding transmission spectra similar to those observed in the experiments [5,18-20]. The paper concludes with a brief summary and a short discussion of the role which the class of the CNTs may play in their applications.

\section{SPECTRUM AND SYMMETRIES OF INFINITE CNTS}

The nearest-neighbor tight-binding CNT Hamiltonian, with one $p_{z}$ electron per atom, reads

$$
\hat{H}=\sum_{\vec{R}} \sum_{\vec{d}} t_{\vec{R}, \vec{R}+\vec{d}}|\vec{R}, A\rangle\langle\vec{R}+\vec{d}, B|+\text { H.c. },
$$

where $\vec{R}$ are the lattice vectors and $\vec{d} \in\left\{0, \vec{a}_{1}, \vec{a}_{2}\right\}$ [cf. Fig. 1(b)]. For simplicity, the spin degree of freedom and spin-orbit interaction effects will be discussed later. The hopping integrals $t_{\vec{R}, \vec{R}+\vec{d}}$ depend on the CNT's curvature [21,22]. In infinite CNTs, we can define the sublattice states $|\vec{k}, p\rangle=$ $1 / \sqrt{N} \sum_{\vec{R}} \exp (i \vec{k} \cdot \vec{R})|\vec{R}, p\rangle$ and obtain the Hamiltonian in the reciprocal space. Introducing

$$
\sum_{\vec{d}} t_{\vec{R}, \vec{R}+\vec{d}} e^{i \vec{k} \cdot \vec{d}}=|\gamma(\vec{k})| \exp [i \eta(\vec{k})],
$$

the eigenstates of $\hat{H}$ are the Bloch states

$$
|\vec{k}\rangle=\frac{1}{\sqrt{2}}\left(e^{i \eta(\vec{k}) / 2}|\vec{k}, A\rangle \pm e^{-i \eta(\vec{k}) / 2}|\vec{k}, B\rangle\right),
$$

with energy $\pm|\gamma(\vec{k})|$, where the $(+/-)$ sign applies to the conduction/valence states, respectively.

The CNT symmetries defining the eigenstates and relevant for us $\left[\mathcal{C}_{\mathfrak{n}}, \mathcal{U}, \mathcal{T}(\vec{T}), \mathcal{S}(\alpha, h)=\mathcal{T}(\vec{H})\right]$ are illustrated for the rolled $(8,2)$ CNT in Fig. 1(a); their characteristic vectors $\vec{T}, \vec{C}_{h}, \vec{H}$ on the graphene plane are shown in Fig. 1(b) and listed in Table I.

The geometry of a CNT is determined by its chiral vector defining the nanotube's circumference [cf. Figs. 1(a) and 1(b)]. 

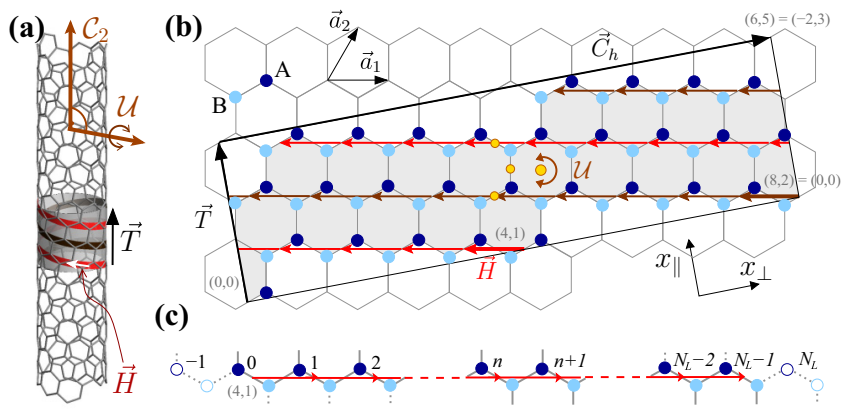

(c)

FIG. 1. (Color online) Real-space structure of a chiral $(8,2)$ nanotube. (a) Fragment of a rolled CNT. The translational unit cell with $2 N_{B}$ atoms is marked in gray; fragments of the two helices constituting the CNT are shown in red and brown. (b) Unrolled translational unit cell. A rotation by $\pi$ around one of the $\mathcal{U}$ axes (some choices of $\mathcal{U}$ are marked with yellow dots) maps all atoms onto those from the opposite sublattice. (c) An unrolled helical chain from an $(8,2)$ CNT of length $L=N_{t}|\vec{T}|$. It has $N_{L}=N_{t} N_{B} / \mathfrak{n}$ cells, where $\mathfrak{n}=\operatorname{gcd}(8,2)=2$. Saturated bonds are shown in solid lines; dangling bonds are shown in dotted lines. "Missing" atoms are marked by open circles.

The chiral vector is given by the chiral indices $\left(m_{1}, m_{2}\right): \vec{C}_{h}=$ $m_{1} \vec{a}_{1}+m_{2} \vec{a}_{2}$ [1,14,23-25]. Let us define $\mathfrak{n}=\operatorname{gcd}\left(m_{1}, m_{2}\right)$; then if a CNT with indices $\left(m_{1} / \mathfrak{n}, m_{2} / \mathfrak{n}\right)$ is metallic, i.e., $\left(m_{1}-m_{2}\right) / \mathfrak{n}=\left.0\right|_{\bmod 3}$, the original $\left(m_{1}, m_{2}\right) \mathrm{CNT}$ is of the (A) class, otherwise it is of the $(Z)$ class. The chiral indices fully determine the symmetries of the CNT $[1,23,25]$, their associated quantum numbers, and the nature of the CNT's eigenstates.

The quantum numbers used to identify a Bloch state are commonly derived either from the pair of symmetries $[\mathcal{C}, \mathcal{T}(\vec{T})]$ (translational scheme) or $\left[\mathcal{C}_{\mathfrak{n}}, \mathcal{S}(\alpha, h)\right]$ (helical scheme), resulting either in the quantum numbers $\left(k_{\perp}, k_{\|}\right)$or $(m, k)$, respectively. We will use mostly the $(m, k)$ pair. The rotational symmetries give rise to the quantized transverse quantum numbers: $\mathcal{C}$ to $k_{\perp}$ and $\mathcal{C}_{\mathfrak{n}}$ to the crystal angular momentum $m$. The one-dimensional (1D) low-energy electronic dispersion is hyperbolic, with minima at Dirac points shifted by the curvature, $\pm \vec{K}_{c}$ [cf. Figs. 2(a) and 2(c)] [21,22]. The Dirac momenta $\pm \vec{K}_{c}$ correspond in the helical representation to $\left( \pm m, \pm K_{c}\right)$, with $m \neq 0$ in CNTs of the (Z) class. In (A)-class CNTs, both valleys share the same crystal angular momentum, $m=0$ [14]. Appendix A contains a short review of the CNT symmetries and the associated structure of the reciprocal space. (a)

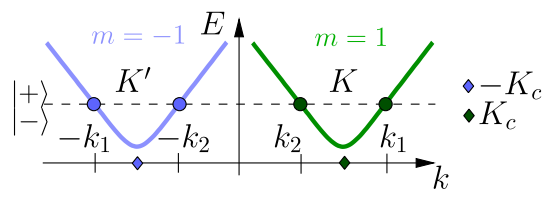

(b)

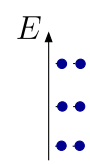

(c)

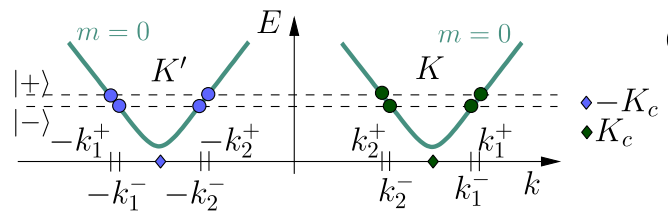

(d)

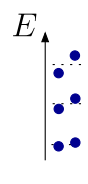

FIG. 2. (Color online) Low-energy conduction spectrum (without spin) of finite CNTs in the helical approach. (a) Energy subbands in a (Z)-class $(6,3)$ CNT. The four degenerate Bloch states of an infinite CNT (points) can be combined into two degenerate valley $\left(K / K^{\prime}\right)$ or $\mathcal{U}$ parity $(+/-)$ eigenstates of a finite-length CNT. The $K / K^{\prime}$ valley corresponds to $m=+1 /-1$, respectively. (b) Part of the doubly degenerate spectrum of a finite (Z)-class CNT. (c) In finite (A)-class CNTs, the $\mathcal{U}$ eigenstates originate from Bloch states with $\vec{k}^{+} \neq \vec{k}^{-}$. (d) The $\mathcal{U}$ pairs in the resulting spectrum are nondegenerate.

Every CNT is also symmetric under a rotation by $\pi$ around an axis $\mathcal{U}$ perpendicular to the $\mathcal{C}$ axis and intersecting either the center of a hexagon or a C-C bond [24] [cf. Figs. 1(a) and 1(b)]. The atomic orbitals and Bloch states transform under $\mathcal{U}$ as $\mathcal{U}|\vec{R}, p\rangle=|-\vec{R},-p\rangle, \mathcal{U}|\vec{k}\rangle=|-\vec{k}\rangle$. In real space, $\mathcal{U}$ involves an exchange of sublattices; in reciprocal space, because $\vec{K}^{\prime}=-\vec{K}$, it involves an exchange of Dirac points (valleys).

\section{FINITE NANOTUBES}

The spectra of the finite nanotubes can be found numerically, by direct diagonalization of the spinless Hamiltonian (1) for a finite lattice. We have performed this diagonalization, including the curvature effects [21,22], with the hopping integrals between $\pi$ and $\sigma$ orbitals set to $V_{\pi}=-2.66$ and $V_{\sigma}=6.38 \mathrm{eV}$ [26]. In the following, we present our analytical derivation of the spectra together with the numerical results.

Unlike the translational and helical symmetries, neither the rotational symmetries $\mathcal{C}, \mathcal{C}_{\mathfrak{n}}$ nor the $\mathcal{U}$ symmetry are broken in finite nanotubes. Thus we can use them to identify the Hamiltonian eigenstates. We construct these eigenstates as linear combinations of Bloch states (3) with the same energy $|\gamma(\vec{k})|$, imposing on them the symmetry constraints and the boundary conditions. The most general $\mathcal{U}$-symmetric

TABLE I. Symmetry operations of an infinite nanotube with chiral indices $\left(m_{1}, m_{2}\right)$, in 3D and 2D. All 2D operations have to be taken modulo $\vec{C}_{h} . N_{B}=2\left(m_{1}^{2}+m_{1} m_{2}+m_{2}^{2}\right) / d_{R}$, where $d_{R}=\operatorname{gcd}\left(2 m_{1}+m_{2}, m_{1}+2 m_{2}\right)$, is the number of graphene unit cells in the translational CNT unit cell. In the helical scheme, $\mathfrak{n}=\operatorname{gcd}\left(m_{1}, m_{2}\right)$, and the screw operation parameters are $\alpha=H_{\perp} / R$ and $h=H_{\|}$. The helical vector $\vec{H}=\left(h_{1}, h_{2}\right)$ can be found from the condition $h_{1} m_{2}-h_{2} m_{1}= \pm \mathfrak{n}[14,23,25]$.

\begin{tabular}{|c|c|c|c|c|c|}
\hline Symmetry & $3 \mathrm{D}$ & $2 \mathrm{D}$ & Quantum numbers & Reciprocal space & Action on $|\vec{k}\rangle$ \\
\hline Rotation by $2 \pi(\mathcal{C})$ & $\mathcal{R}(2 \pi)$ & $\mathcal{T}\left(\vec{C}_{h}\right)$ & $k_{\perp}=\vec{k} \cdot \vec{C}_{h} / C_{h}$ & $\begin{array}{l}k_{\perp}=l_{\perp} / R, \\
l_{\perp} \in\left(N_{B} / 2, N_{B} / 2\right]\end{array}$ & $\mathcal{T}\left(\vec{C}_{h}\right)|\vec{k}\rangle=|\vec{k}\rangle$ \\
\hline Translation by $\vec{T}$ & $\mathcal{T}(\vec{T})$ & $\mathcal{T}(\vec{T})$ & $k_{\|}=\vec{k} \cdot \vec{T} / T$ & $k_{\|} \in(-\pi / T, \pi / T]$ & $\mathcal{T}(\vec{T})|\vec{k}\rangle=e^{i k_{\|} T}|\vec{k}\rangle$ \\
\hline Rotation by $2 \pi / \mathfrak{n}\left(\mathcal{C}_{\mathfrak{n}}\right)$ & $\mathcal{R}(2 \pi / \mathfrak{n})$ & $\mathcal{T}\left(\vec{C}_{h} / \mathfrak{n}\right)$ & $m=\vec{k} \cdot \vec{C}_{h}$ & $m \in[0, \mathfrak{n}-1]$ & $\mathcal{T}\left(\vec{C}_{h} / \mathfrak{n}\right)|\vec{k}\rangle=e^{i 2 \pi m / \mathfrak{n}}|\vec{k}\rangle$ \\
\hline Screw (helical) & $S(\alpha, h)$ & $\mathcal{T}(\vec{H})$ & $k=\vec{k} \cdot \vec{H} / H$ & $k \in(-\pi / h, \pi / h]$ & $\mathcal{T}(\vec{H})|\vec{k}\rangle=e^{i(m \alpha+k h)}|\vec{k}\rangle$ \\
\hline Rotation by $\pi$ around the $\mathcal{U}$ axis & $\mathcal{U}$ & $\mathcal{U}$ & $+/-$ & & $\mathcal{U}|\vec{k}\rangle=|-\vec{k}\rangle$ \\
\hline
\end{tabular}


eigenstate is a combination of the four momentum states with the same $|\gamma(\vec{k})|$ [see Figs. 2(a) and 2(c)],

$$
|\psi\rangle=\sum_{i=1,2}\left|a_{i}\right|\left(e^{i \beta_{i}}\left|m, k_{i}\right\rangle+e^{-i \beta_{i}}\left|-m,-k_{i}\right\rangle\right) .
$$

We denote the four momenta by $\left( \pm \vec{k}_{1}, \pm \vec{k}_{2}\right)$, with $+\vec{k}$ in the $K$ valley and $-\vec{k}$ in $K^{\prime}$; the index "1" stands for $|k|>K_{c}$ and "2" stands for $|k|<K_{c}$. In a CNT of length given by $\vec{L}=N_{t} \vec{T}$, the $\mathcal{U}$ axis is at $\left(\vec{C}_{h}+\vec{L}-\vec{H}\right) / 2$, with $\vec{H}$ the helical vector. Since the energy eigenstates should also be eigenstates of $\mathcal{U}$, $\mathcal{U}\left|\psi^{ \pm}\right\rangle:= \pm\left|\psi^{ \pm}\right\rangle$, the phases $\beta_{i}$ must obey

$$
\vec{k}_{i} \cdot(\vec{L}-\vec{H})+2 \pi m n_{\alpha} / \mathfrak{n}+2 \beta_{i}=n_{i} \pi, \quad n_{i} \in \mathbb{Z} .
$$

The integers $n_{i}$ are even (odd) for even (odd) $\mathcal{U}$ eigenstates, which ensures their mutual orthogonality. Note that a priori the even and odd states can also have different momenta, $\vec{k}_{i}^{ \pm}$. The factor $2 \pi n_{\alpha} / \mathfrak{n}$ accounts for the difference between the angular coordinates of the end and the beginning of a helical chain.

The finite nature of the system is encoded in the boundary conditions. We require that the wave functions should vanish at some points, to be specified later, beyond the boundary sites.

\section{A. Boundary conditions: Zigzag class}

The usual boundary conditions applied in pure zigzag ribbons and CNTs [22,27] are $\psi_{A}\left(\vec{R}_{L}\right)=0 \wedge \psi_{B}\left(\vec{R}_{R}\right)=0$, with $\vec{R}_{L}$ and $\vec{R}_{R}$ the first "missing" lattice sites beyond the left and right CNT ends, respectively [see Fig. 1(c)]. Note that the $\mathcal{U}$ symmetry already implies $\psi_{A}\left(\vec{R}_{L}\right)=\psi_{B}\left(\vec{R}_{R}=\right.$ $\left.\vec{L}+\vec{C}_{h}-\vec{H}-\vec{R}_{L}\right)$; the finite size of the system in addition sets this value to 0 . When applied to (Z)-class CNTs, this yields

$$
\sin \left[\left(\vec{k}_{1}-\vec{k}_{2}\right) \cdot \vec{R}_{L}^{\prime}+\left(\eta_{1}-\eta_{2}\right) / 2\right]=0,
$$

with $\vec{R}_{L}^{\prime}=\vec{R}_{L}-(\vec{L}-\vec{H}) / 2$. The left side of (6) defines a "quantization function" whose zeros yield discrete momentum values, corresponding to discrete longitudinal $\left(k_{\|}\right.$or $\left.k\right)$ modes. The solutions of (6) determine the momentum pairs $\vec{k}_{1}(n), \vec{k}_{2}(n)$, where $\vec{k}_{i}(n)=\vec{k}_{i}^{+}(n)=\vec{k}_{i}^{-}(n)$. Out of each pair, with appropriate $\beta_{i}$ 's, we can construct two sets of degenerate states: either $\mathcal{U}+/-$ states or valley $K / K^{\prime}$ states. The $\mathcal{U}$ eigenstates are

$$
| \pm, n\rangle_{Z}=\frac{1}{2} \sum_{j=1,2}\left|a_{j}\right| \sum_{\tau= \pm} f^{ \pm}(\tau) e^{i \tau \beta_{j}}\left|\tau m, \tau k_{j}(n)\right\rangle,
$$

where $f^{+}(\tau)=1$ and $f^{-}(\tau)=-i \tau$. The phases $\beta_{j}$ fulfill the condition (5), and we have chosen here $\beta_{j}^{-}=\beta_{j}^{+}-\pi / 2$, with $\beta_{j}:=\beta_{j}^{+}$. The valley states are linear combinations of $\mathcal{U}$ states,

$$
\left|K / K^{\prime}, n\right\rangle_{Z} \equiv| \pm m, n\rangle_{Z}=\frac{1}{\sqrt{2}}\left(|+, n\rangle_{Z} \pm i|-, n\rangle_{Z}\right) .
$$

The energy spectrum consists of doubly degenerate shells [cf. Fig. 2(b)], numbered by $n$. The level spacing between consecutive shells resulting from (6) varies with energy, as shown in Fig. 3(c).

In the helical scheme, the natural choice of the vanishing sites is at $-\vec{H}$ and at $\vec{L}$ from the origin of each helical chain, as shown in Fig. 1(c). The translational unit cell of a $(6,3)$ (a)
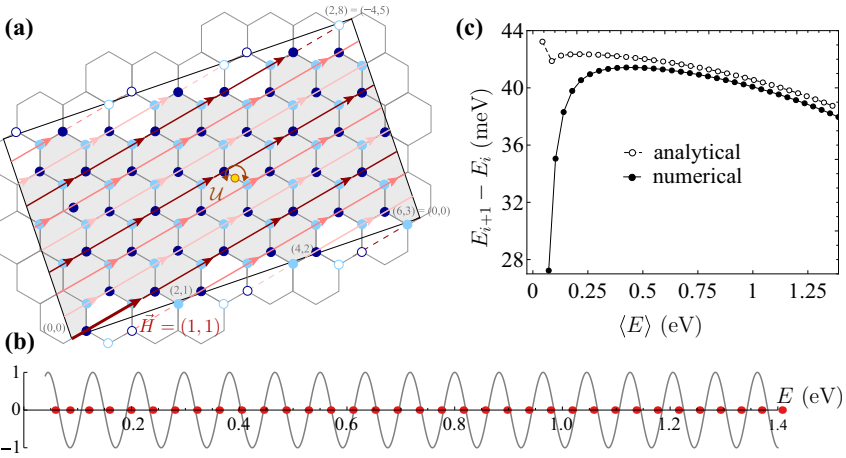

FIG. 3. (Color online) (a) The translational unit cell of a $(6,3)$ zigzaglike CNT. The helical vector is $\vec{H}=(1,1)$ and the CNT is constructed from three helical chains. (b) The quantization function for a $(6,3) \times 36 \mathrm{CNT}$; its zeros define the energy levels of the nanotube. The points on the horizontal axis are the numerical eigenvalues of the Hamiltonian (1) on the finite lattice. (c) The spacing between consecutive energy shells, numerical and analytical. The initially bad agreement improves with the distance from the CNP.

CNT is shown in Fig. 3(a), with its three helical chains and the vector $\vec{H}=(1,1)$. The "missing" atoms where the wave functions are set to vanish are marked in open circles. These boundary conditions yield the momentum quantization, with $\vec{R}_{L}=-\vec{H}$ :

$$
\sin \left[-\left(\vec{k}_{1}-\vec{k}_{2}\right) \cdot(\vec{L}+\vec{H}) / 2+\left(\eta_{1}-\eta_{2}\right) / 2\right]=0 .
$$

The course of the quantization function for a $(6,3) \times 36 \mathrm{CNT}$ [i.e., a $(6,3)$ CNT with 36 unit cells] is plotted in Fig. 3(b). The zeros of this function do not fully coincide with the numerical results, but their qualitative features are similar. In both, the energy spectrum is doubly degenerate and the spacing between the energy levels varies with the distance from the charge neutrality point (CNP). This variation, shown in Fig. 3(c), at low energies differs between numerics and analytics, recovering a very good agreement at higher energies.

The same quantization condition (6) can be obtained by exploiting the $\mathcal{C}_{\mathfrak{n}}$ symmetry of the nanotube, which is not broken by the edges. The constraint on the wave function is now that it must vanish on the ends of each of the helical chains, $\psi_{A}\left(\vec{R}_{L}\right)=\psi_{A}\left(\vec{R}_{L}+n \vec{C}_{h} / \mathfrak{n}\right)=0$, with $n=1, \ldots, \mathfrak{n}-1$. Because the $\pm \vec{k}$ components of the wave function have phase differences of $\pm 2 \pi m / \mathfrak{n}$ between neighboring chains, these $\mathfrak{n}-1$ conditions can be satisfied only if (6) is.

At low energies (before the trigonal warping comes into play), Eq. (9) becomes

$$
\kappa_{\perp}=\kappa_{\|} \cot \left[\kappa_{\|}(L+h)\right],
$$

with $\kappa_{\|}=k_{1 \|}-K_{\|}^{c}=K_{\|}^{c}-k_{2 \|}$ and $\kappa_{\perp}=k_{1 / 2 \perp}-K_{\perp}^{c}$. This relation, shown here in the translational scheme in which it usually is derived, is the standard momentum quantization condition for pure zigzag CNTs [22,27].

\section{B. Boundary conditions: Armchair class}

In the (A)-class CNTs, the low-energy states belong to the $m=0$ subband. The constraint $\psi_{A}\left(\vec{R}_{L}\right)=0=\psi_{B}\left(\vec{R}_{R}\right)$ alone is not sufficient and we must constrain both sublattices at both ends. It is enough to impose that both $\psi_{A}$ and $\psi_{B}$ must 
vanish on the left end of the CNT, at positions $\vec{R}_{L, A}$ and $\vec{R}_{L, B}$, respectively - their vanishing on the right follows from the $\mathcal{U}$ symmetry. The set of equations $\psi_{A}\left(\vec{R}_{L, A}\right)=0=\psi_{B}\left(\vec{R}_{L, B}\right)$ yields a momentum quantization condition,

$$
\begin{aligned}
0= & \sin \left[\left(\eta_{1}^{\prime}+\eta_{2}^{\prime}\right) / 2\right] \sin \left[\left(\vec{k}_{1}-\vec{k}_{2}\right) \cdot \vec{R}_{L}^{\prime}\right] \\
& \pm \sin \left[\left(\eta_{1}^{\prime}-\eta_{2}^{\prime}\right) / 2\right] \sin \left[\left(\vec{k}_{1}+\vec{k}_{2}\right) \cdot \vec{R}_{L}^{\prime}\right],
\end{aligned}
$$

where $\vec{R}_{L}^{\prime}=\left(\vec{R}_{L, A}+\vec{R}_{L, B}-\vec{L}-\vec{H}\right) / 2$ and $\eta_{i}^{\prime}=\eta_{i}-\vec{k}_{i}$. $\left(\vec{R}_{L, A}-\vec{R}_{L, B}\right)$. In contrast to the (Z)-class case, the quantization function now contains two terms. The first originates from the intravalley, while the second comes from the intervalley backscattering. The presence of the latter can be traced back to the nonvanishing value of the intervalley scalar product, $\langle\vec{k} \mid-\vec{k}\rangle \neq 0$. In the (Z)-class CNTs, $\langle\vec{k} \mid-\vec{k}\rangle=0$; the orthogonality of opposite valley states is protected by the $\mathcal{C}_{\mathfrak{n}}$ symmetry.

The $+/-$ sign in the second line of (11) refers to even/odd $\mathcal{U}$ eigenstates, resulting in different quantization for $\vec{k}^{+}$and $\vec{k}^{-}$ and, hence, in the energy splitting

$$
\Delta_{+-}(n)=E\left(\vec{k}^{+}(n)\right)-E\left(\vec{k}^{-}(n)\right) .
$$

The nondegenerate $\mathcal{U}$ eigenstates can be written as

$$
| \pm, n\rangle_{A}=\frac{1}{2} \sum_{j=1,2}\left|a_{j}^{ \pm}\right| \sum_{\tau= \pm} e^{i \tau \beta_{j}^{ \pm}}\left|0, \tau k_{j}^{ \pm}(n)\right\rangle,
$$

where both $\beta_{j}^{ \pm}$are now defined by (5) for different momenta $\vec{k}_{j}^{ \pm}$. Note that the transformation which led from (7) to (8) now yields only approximate valley states, where the other valley is strongly suppressed, but since $k^{+} \neq k^{-}$, the suppression cannot be complete.

The quantization function for an $(8,2) \times 58 \mathrm{CNT}$ with one choice of $\vec{R}_{L, A}, \vec{R}_{L, B}$ [Fig. 4(a)] is shown in Fig. 4(b).

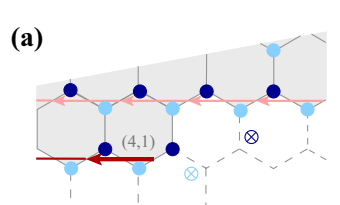

(c)

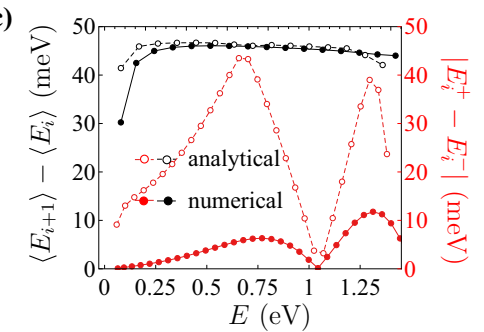

(b)

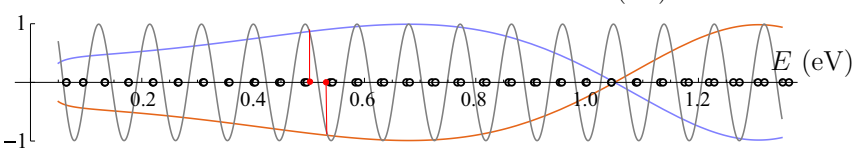

FIG. 4. (Color online) (a) The neighborhood of the left end of the helical chain starting at lattice site $(4,1)$. The wave function vanishes at the points marked by the circled crosses. (b) The quantization function (11). The first term is plotted in gray; the opposite of the second term for even/odd eigenstates is plotted in orange/blue. The energy levels are given by the intersections of the gray line with the colored ones, as indicated by the red dots depicting a pair of analytical solutions near $E=0.5 \mathrm{eV}$. Numerical energy eigenvalues are marked by the open circles on the horizontal axis. (c) The distance between mean shell energies $\left\langle E_{i+1}\right\rangle-\left\langle E_{i}\right\rangle=\left(E_{i+1}^{+}+E_{i+1}^{-}\right) / 2-\left(E_{i}^{+}+E_{i}^{-}\right) / 2$ is shown in black. The energy spacing within the shells, $\Delta_{+-}=\left|E_{i}^{+}-E_{i}^{-}\right|$, is shown in red.
The positions of the energy levels do not match well with the numerical results, but other features show qualitative agreement. The spectrum consists of a set of nearly degenerate doublets, with energies $E_{i}^{+}$and $E_{i}^{-} ; i$ denotes the number of the doublet when reckoned from the CNP and $+/-$ is the even/odd $\mathcal{U}$ parity eigenstate. The variation of the spacing between mean doublet energies $\left|\left(E_{i+1}^{+}+E_{i+1}^{-}\right) / 2\right|-\left|\left(E_{i}^{+}+E_{i}^{-}\right) / 2\right|$ [black in Fig. 4(c)] is similar for numerical and analytical results. The energy splitting within the doublets, $\Delta_{+-}=\left|E_{i}^{+}-E_{i}^{-}\right|$[red in Fig. 4(c)], also varies with the distance from the CNP, though the analytical solution seriously overestimates the splitting. One possible method to cure the discrepancy between the analytical and numerical results would be to allow $\vec{R}_{L, A}$ and $\vec{R}_{L, B}$ to vary with energy, reflecting a weaker confinement of the higher-energy states.

The Fourier transform of obtained eigenstates shows that the even and odd states indeed have different momenta, $\vec{k}_{i}^{+}$and $\vec{k}_{i}^{-}$, whose difference yields $\left|\Delta_{+-}\right| \simeq \hbar v_{F}\left|k_{i}^{+}-k_{i}^{-}\right|$, as shown in Fig. 5(a).

The value of $\Delta_{+-}$naturally decreases with the length of the CNT [see Fig. 5(b)], since it is a finite-size effect. It also oscillates with growing amplitude and frequency as the shell number increases, which may be due to the confinement varying with energy.

The structure of Eq. (11) suggests that for some solutions $\Delta_{+-}(E)$ is 0 , and these solutions should correspond to $\vec{k}_{i}$. $\vec{R}_{L}^{\prime}=p_{i} \pi / 2$. Since the dispersion is nearly linear, the zeros of $\Delta_{+-}(E)$ should lie approximately on hyperbolae in the $(E, L)$ plane or on straight lines in the $(E, 1 / L)$ plane. As can be seen in Fig. 5(c), the numerical zeros indeed follow this prediction rather well.

We close this section with three remarks on the relation of our work to that of other authors.

First, for a pure armchair, Eq. (11) also holds, but because there $\eta_{1} \equiv \pi$ and $\eta_{2} \equiv 2 \pi$, the eigenstates (4) contain either only $\pm \vec{k}_{1}$ or only $\pm \vec{k}_{2}$. The result is a quantum box quantization, $2 \vec{k} \cdot \vec{R}_{L}^{\prime}=n \pi[8]$.

Second, we notice that in several previous works, both in CNTs [15] and in graphene nanoribbons [17], boundary conditions were introduced as a reflection matrix $M$ that links left and right movers on the boundary and results were delivered based on this ansatz. Our work is complementary to these: we begin with a given atomic structure from which the precise form of the reflection matrix in the valley space can be obtained. Thus it provides a well-defined base for further studies.

Third, in experimental devices, the CNT quantum dots are usually defined electrostatically by the gates. Our numerical modeling of CNTs in soft confining potentials with preserved $\mathcal{C}_{\mathfrak{n}}$ symmetry (see Appendix B) shows that the spectra are then modified, but still clearly belong either to the $(\mathrm{A})$ or $(\mathrm{Z})$ class.

\section{INCLUSION OF THE SPIN-ORBIT INTERACTION (SOI)}

The SOI breaks both the $\mathcal{U}$ symmetry and the degeneracy between the states $|\vec{k}, \sigma\rangle$ and $|-\vec{k}, \sigma\rangle$ [22]. The time-reversal and $\mathcal{C}_{\mathfrak{n}}$ symmetries are preserved and the spectrum consists of a series of Kramers' doublets. For (Z)-class CNTs, the shell quantization still follows from (6), now with spin-dependent 

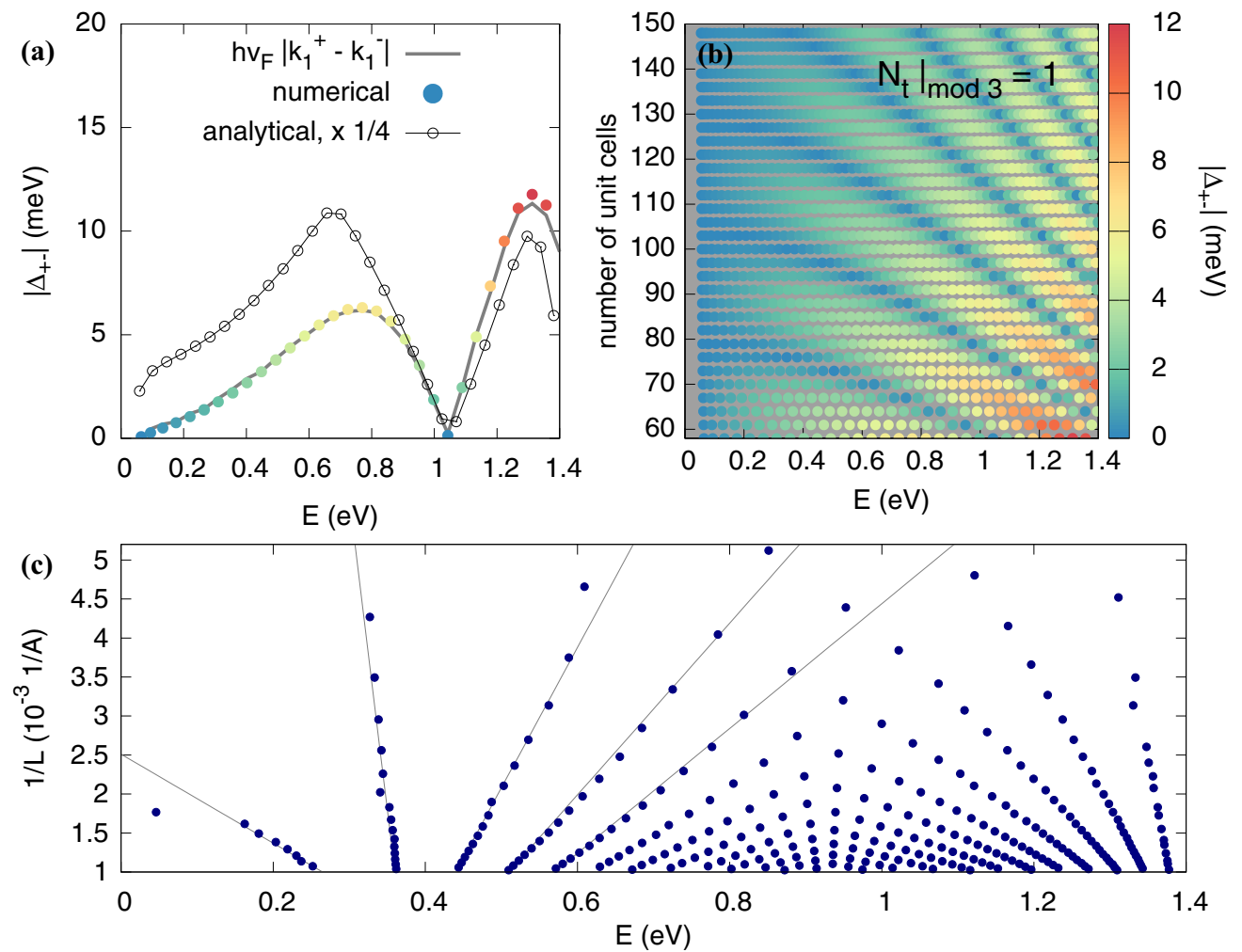

FIG. 5. (Color online) Spectral properties of finite $(8,2)$ CNTs without spin-orbit coupling. (a) The splitting $\left|\Delta_{+-}\right|$vs mean shell energy for an $(8,2) \times 58$ nanotube, matching the numerically computed $\hbar v_{F}\left|k_{i}^{+}-k_{i}^{-}\right|$. (b) The dependence of $\left|\Delta_{+-}\right|$(numerical) on the length of the CNT and the mean shell energy, for $\left.N_{t}\right|_{\bmod 3}=1$. The values for $N_{t}=58$ correspond to the $\Delta_{+-}(E)$ plotted in (a). (c) The dots mark the positions of shells with $\Delta_{+-}(E)=0$ for CNTs with lengths from 30 to 150 unit cells. The vertical axis is chosen as $1 / L$ in order to emphasize the hyperbolic dependence of the position of zeros of $\Delta_{+-}(E)$ on $L$ (and, hence, linear dependence on $1 / L$ ). The lines have been drawn as a guide to the eye.

$\eta(\vec{k}, \sigma)$ [22]. The doublets are $\left\{|K, \sigma\rangle,\left|K^{\prime},-\sigma\right\rangle\right\}$, split by the energy scale $\Delta_{S O}$. In (A)-class CNTs, the eigenstates are more complex. The Kramers' pairs are $\{|\alpha, \uparrow\rangle,|\beta, \downarrow\rangle\}$ and $\{|\gamma, \downarrow\rangle,|\delta, \uparrow\rangle\}$, where $|\alpha\rangle,|\beta\rangle,|\gamma\rangle,|\delta\rangle$ denote combinations of valley states, determined by the orthogonality requirements $\langle\alpha \mid \delta\rangle=0=\langle\beta \mid \gamma\rangle$. The splitting $\Delta$ between Kramers' pairs is larger than $\Delta_{S O}$ (cf. Fig. 6).

The finite $\Delta_{S O}$ makes it practically impossible to distinguish between the two spectral classes in a spectroscopic experiment. Nevertheless, $\Delta_{+-}$reemerges in the presence of a finite parallel magnetic field $B_{\|}$. This field breaks the Kramers' degeneracy; the four levels in one shell evolve differently with varying field magnitude (see Fig. 6). At some value $\left(B_{S O}\right)$ of $B_{\|}$, the acquired Aharonov-Bohm phase compensates $\Delta_{S O}$ [22] for one spin direction, locally restoring the $\mathcal{U}$ symmetry. There we expect $\Delta_{+-}$to become apparent in (A)-class CNTs. We have tested this hypothesis with numerical calculations of the transmission through finite nanotubes with spin-orbit coupling in $B_{\|}$using Green's function techniques [28-30]. The results are shown in Fig. 6. Initially, at $B_{\|}=0$, the spectra of both CNTs classes are similar. At $B_{\|}=B_{S O}$ in (Z)-class CNTs, the $K / K^{\prime}$ states with spin $\downarrow$ merely cross, while in the (A)-class CNTs, the $\Delta_{+-}$split manifests as an anticrossing. This effect is present in all shells throughout the low-energy spectrum (not shown).

The signature of a valley mixing has been seen in several transport experiments on ultraclean CNT quantum dots in magnetic field [5,18-20] and attributed to the presence of weak disorder. We studied the impact of an on-site disorder on the CNT spectra and show the results in Appendix C. The disorder introduces a level splitting in all CNTs. In the (Z)-class CNTs, this is the only mechanism removing the level degeneracy;
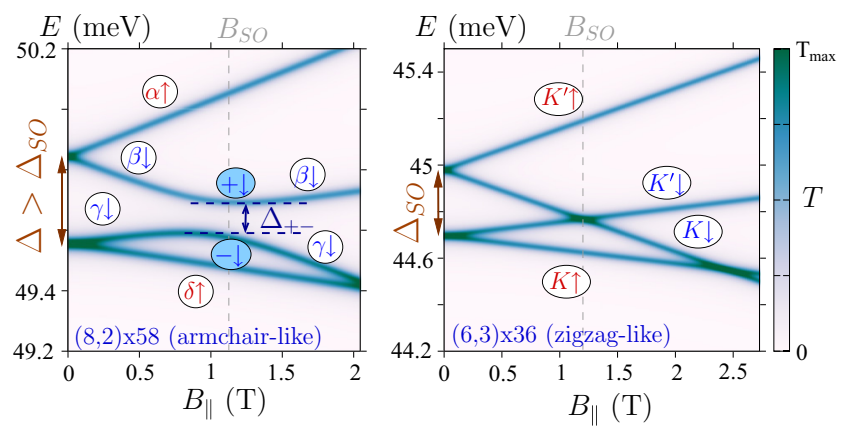

FIG. 6. (Color online) The transmission of finite, disorder-free nanotubes in varying parallel magnetic field $B_{\|}$, in an energy range corresponding to one shell. The orbital degeneracy is removed by the SOI also in (Z)-class CNTs and two Kramers' pairs split by $\Delta_{S O}$ are present at $B_{\|}=0$. In (A)-class CNTs, the splitting at zero field $\Delta$ is larger than $\Delta_{S o}$, due to the boundary effects. At $B_{\|} \neq 0$, the time-reversal symmetry is broken. The four CNT levels evolve differently: in (A)-class CNTs, when $B_{\|}=B_{S O}$, the splitting $\Delta_{+-}$ manifests as an anticrossing. In (Z)-class CNTs, the valley states remain independent. 
in the (A) class, it adds to the native $\Delta_{+-}$. The disorderinduced split varies for different realizations of disorder (e.g., if the quantum dot is shifted along the CNT), while the $\Delta_{+-}$does not; this allows for their unambiguous separation. Nevertheless, Fig. 9 in Appendix C suggests a simpler method: smooth changes of the measured anticrossing with the shell energy can be taken as an indication of dominant $\Delta_{+-}$.

\section{CONCLUSIONS}

Every finite CNT belongs to one of the two spectral classes. One is the $(Z)$ class, with a degenerate spectrum and the $+m /-m$ value of crystal angular momentum associated to the $K / K^{\prime}$ valley. The (A)-class CNTs have nondegenerate spectrum and their lowest-energy states all have $m=0$. The impossibility of removing the valley mixing in (A)-class CNTs can be crucial, both in the attempts to use them for quantum computing, relying on the manipulation of spin and valley [7,31], and in the search for Majorana bound states [32]. Because the splitting $\Delta_{+-}$is bound inextricably to the finite size of the system, it is not present in the Hamiltonian of an infinite CNT.

Note added in proof. Recently, we have become aware of a new publication considering the influence of chirality and boundary type on the valley mixing [33].

\section{ACKNOWLEDGMENTS}

We gratefully acknowledge the discussions with Stephan Lochner, Andreas K. Hüttel, and Christoph Strunk. We also thank the DFG for the financial support under Grants No. GRK 1570 and No. SFB 689.

\section{APPENDIX A: CNT SYMMETRIES}

The rolling of the graphene plane corresponds mathematically to imposing the condition $\vec{R} \equiv \vec{R}+\vec{C}_{h}$, where $\vec{C}_{h}=$ $m_{1} \vec{a}_{1}+m_{2} \vec{a}_{2}$ is the chiral vector of the CNT defining its circumference. The nanotube is naturally invariant under rotation by $2 \pi$ around its cylindrical axis-the $\mathcal{C}$ operation. If $\operatorname{gcd}\left(m_{1}, m_{2}\right)=\mathfrak{n} \neq 1$, the CNT is, in addition, symmetric under rotations by $2 \pi / \mathfrak{n}$ around its cylindrical axis $\left(\mathcal{C}_{\mathfrak{n}}\right.$ operations). These rotational symmetries are preserved in finite CNTs.

Each nanotube is a complex periodic system and several schemes have been proposed $[1,23,25]$ for generating the whole lattice out of a smaller unit cell. In the two most common approaches, the CNT is generated either by repeated application of translations by a vector $\vec{T}$, with the unit cell spanned by $\vec{C}_{h}$ and $\vec{T}$ (the linear-angular approach from Ref. [25]), or by repeated application of screw translations $\mathcal{S}(\alpha, h)$ (rotation by $\alpha$ around and translation by $h$ along the $\mathcal{C}$ axis) and $\mathcal{C}_{\mathfrak{n}}$ rotations to the diatomic cell of graphene (helical-angular approach). In the former scheme, a nanotube is decomposed into a linear chain with the unit cell containing many atoms; in the latter approach, it becomes a set of $\mathfrak{n}$ helical chains, each with a diatomic unit cell. These two schemes are illustrated in Figs. 1(a) and 1(b).

In the reciprocal space, the two approaches correspond to two different tilings of the space. In the translational picture, we have $N_{B}=2\left(m_{1}^{2}+m_{1} m_{2}+m_{2}^{2}\right) / d_{R}$ subbands corresponding to different $k_{\perp}$, each of them with $k_{\|} \in$ $(-\pi / T, \pi / T]$. In the helical picture, we have $\mathfrak{n}$ subbands with different values of crystal angular momentum $m$, each of them with $k \in(-\pi / h, \pi / h]$. This is illustrated in Figs. 7(a) and 7(b) for a (Z)-class nanotube and in Figs. 7(c) and 7(d) for an (A)-class nanotube.

$(6,3) \quad$ zigzag class (Z)

(a)

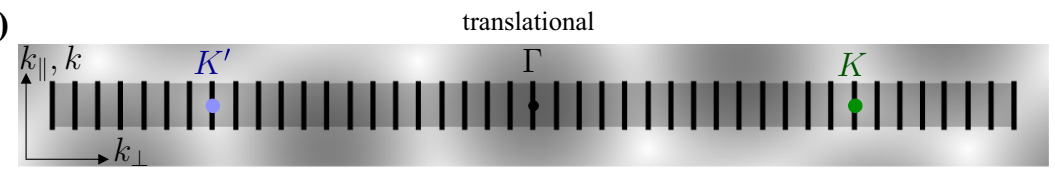

(b)

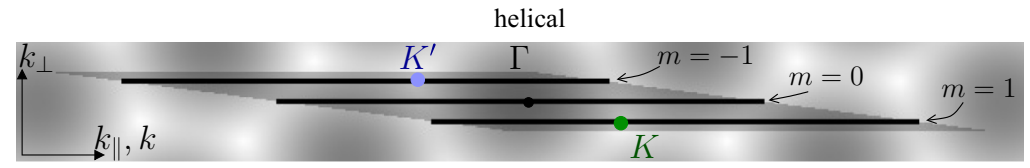

$(8,2)$ armchair class (A)

(c)

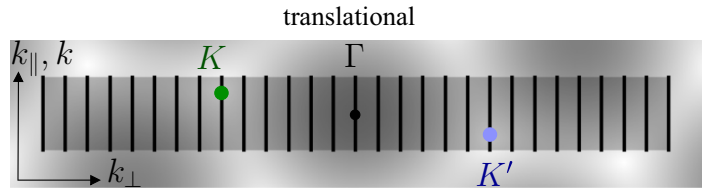

(d)

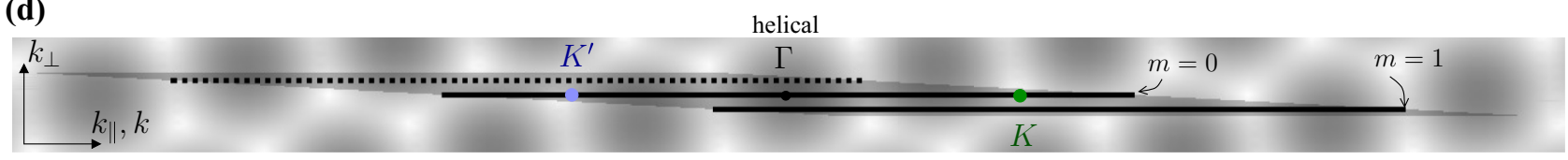

FIG. 7. (Color online) The reciprocal space structure of the two classes of nanotubes. A zigzag class $(6,3)$ is shown in the (a) translational and (b) helical approach. Note that in the translational scheme, both Dirac points lie at $k_{\|}=0$. An armchair-class $(8,2)$ nanotube is shown in the translational approach in (c) and in the helical approach in (d). 


\section{APPENDIX B: SOFT CONFINING POTENTIAL}

So far in our approach, we assumed a sharply defined atomic lattice, with the on-site energies set everywhere to 0 . In transport experiments, however, the nanotubes are usually covered by, or lying across, electric leads - then the ends of the "active" (measured) part of the nanotube are defined electrostatically. The confinement can be shaped further by the use of gate electrodes. A natural question arises as to whether in smooth confining potentials, such as in quantum-dot devices, the difference between (Z)-class and (A)-class spectra will still be present.

We model the smooth confining potential as a modulation of the on-site energy on the atomic sites across the nanotube, $\varepsilon_{0}\left(x_{\|}\right)=V\left(x_{\|}\right)$. The edge atoms have on-site energies equal to the work function of the CNT, which is $\sim 5 \mathrm{eV}$. The potential then falls off smoothly towards the center of the tube. We use two types of potential, i.e., either exponential or polynomial:

$$
\begin{aligned}
& V\left(x_{\|}\right)=V_{0}\left[\exp \left(-\frac{x_{\|}}{\lambda}\right)+\exp \left(-\frac{L-x_{\|}}{\lambda}\right)\right], \\
& V\left(x_{\|}\right)=V_{0}(n)\left[x_{\|}-(L / 2)\right]^{n} .
\end{aligned}
$$

We vary the parameters $\lambda$ and $n$ so as to obtain a range of potentials with slopes of decreasing steepness. Parts of the resulting spectra are shown in Fig. 8 and again separate into two distinct classes. The zigzaglike nanotubes have a spectrum predominantly composed of degenerate doublets, with some
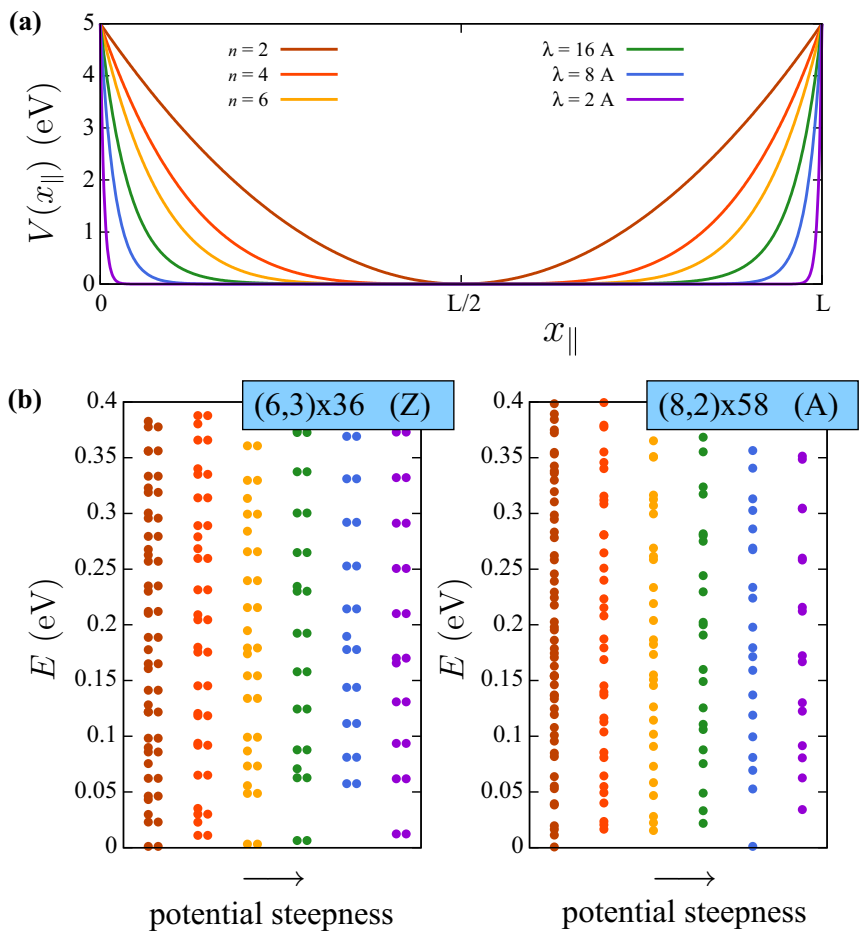

FIG. 8. (Color online) (a) The shape of the confining potential for the parameters used in our numerical calculation. (b) Parts of the spectra of a zigzaglike and an armchairlike CNT in the potentials shown in (a). The points denote the energy levels-most of them doubly degenerate in the $(6,3) \mathrm{CNT}$ and all of them nondegenerate in the $(8,2)$ nanotube. The color of the spectrum points corresponds to the potential in (a) with which the spectrum is obtained. single-energy levels appearing as the potential becomes less steep. Those states are developing in the areas of increased on-site potentials.

The spectra of armchairlike nanotubes remain nondegenerate, though the shape of the potential influences the distances between the energy levels and the splitting between +/pairs. In consequence, the smooth confinement preserves the zigzaglike or armchairlike character of the spectrum.

In non- $\mathcal{U}$-symmetric potentials, the two classes of nanotubes still have two different types of spectra. The crucial symmetry here is the $\mathcal{C}_{\mathfrak{n}}$, since it is the one which defines the crystal angular momentum.

\section{APPENDIX C: DISORDER}

We model the disorder in the nanotube by introducing into the lattice a number of randomly distributed sites with increased on-site energies [34]. The spinless Hamiltonian (1) from the main text then becomes

$$
\begin{aligned}
\hat{H}= & \sum_{\vec{R}_{i}, p_{i}} \epsilon_{i}\left|\vec{R}_{i}, p_{i}\right\rangle\left\langle\vec{R}_{i}, p_{i}\right| \\
& +\sum_{\vec{R}} \sum_{\vec{d}} t_{\vec{R}, \vec{R}+\vec{d}}|\vec{R}, A\rangle\langle\vec{R}+\vec{d}, B|+\text { H.c. }
\end{aligned}
$$

The $\vec{R}_{i}, p_{i}$ denote the disorder sites and $\epsilon_{i}$ is the on-site energy, which we chose to be equal for all sites $i$. We show in Fig. 9 the level splittings in the spectrum of the Hamiltonian $(\mathrm{C} 1)$ for an (A)-class $(8,2) \mathrm{CNT}$ with the length of $\sim 76 \mathrm{~nm}$ (6496 atoms), and a (Z)-class $(6,3) \mathrm{CNT}$ with the length of $\sim 82 \mathrm{~nm}(6048$ atoms). The number of disorder sites is 4 , and their energies are either 1.5 or $0.15 \mathrm{eV}$.

The presence of the disorder, which breaks the $\mathcal{C}_{\mathfrak{n}}$ symmetry, lifts the level degeneracies in the (Z)-class CNTs, with the average splitting increasing as the number of disorder sites or their on-site energy rises. In an (A)-class CNT, we observe a scattered modulation of the original level splittings, usually increased above the values from the pristine nanotube. For on-site energies in the range of $\sim 0.1 \mathrm{eV}$, the disorder effects
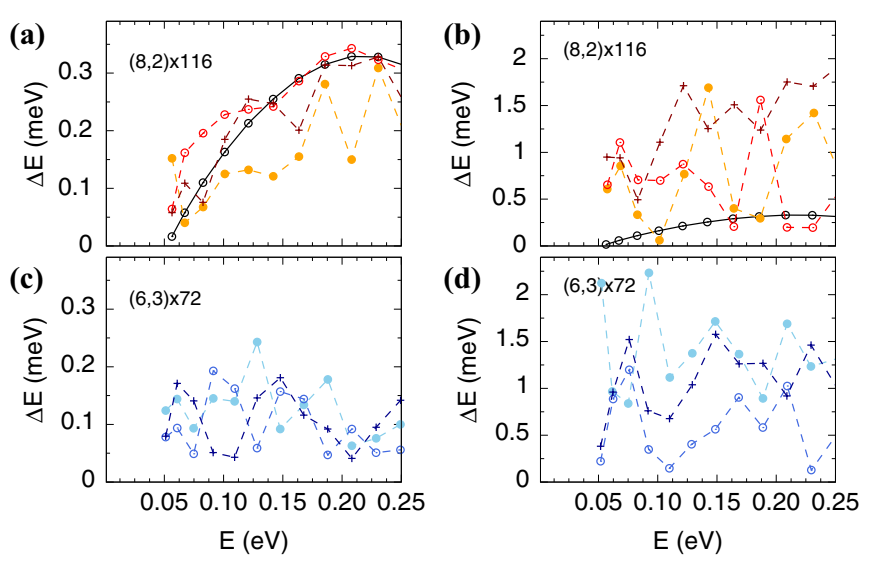

FIG. 9. (Color online) The shell level splitting as a function of the shell energy for (a),(b) an (A)-class (8,2) CNT and (c),(d) a (Z)-class $(6,3) \mathrm{CNT}$ with $N_{i}=4$ disorder sites. The on-site energy of the disorder sites is (a),(c) $0.15 \mathrm{eV}$ or (b),(d) $1.5 \mathrm{eV}$. The three different point types denote the results from three different random configurations of disorder. The solid lines in the plot for the (A)-class CNT denote the shell level spacings in a pristine nanotube. 
are small in comparison with the native $\Delta_{+-}$. For energies in the range of $\sim 1 \mathrm{eV}$, the disorder dominates.

In principle, the disorder in carbon nanotubes [35] can significantly perturb their band structure, e.g., the lattice defects can introduce into the nanotube segments of different chirality. Nevertheless, the valley mixing observed in the experiments $[5,18,20]$ is small, and therefore unlikely to be caused by such strong perturbations. The modeling of the disorder through the variation of the on-site energies should therefore be applicable to describe the experimental situation.

In the experiments on ultraclean CNT quantum dots in magnetic fields, where the valley mixing manifests as a level anticrossing $\Delta_{K K^{\prime}}$, a scan of several energy shells may reveal to which class the CNT belongs. A regular and smooth evolution of $\Delta_{K K^{\prime}}$ with the shell energy would be a clear signature of an (A)-class CNT. A pseudorandom distribution of $\Delta_{K K^{\prime}}(E)$ would indicate the presence of disorder, without ruling out either of the spectral classes. The only experimental investigation of the $\Delta_{K K^{\prime}}$ as a function of the gate voltage in Ref. [18] shows a $\Delta_{K K^{\prime}}(E)$ which is initially scattered and shows a smoother oscillation for higher shells. This indicates that the disorder is present and neither proves nor disproves the presence of a boundary-induced $\Delta_{+-}$.
If the nanotube in the quantum-dot device is ultraclean and valley mixing without clear regular dependence on the shell energy is observed, then the effect of disorder might be disentangled from the boundary-induced $\Delta_{+-}$by changing the position of the quantum dot along the CNT. This can be achieved either by the use of multiple gates [36] or by using as the gate electrode a movable scanning tunneling microscopy (STM) tip [37] at such a distance from the sample which prevents the tunneling current but suffices to modulate the electrostatic potential. While the boundary-induced $\Delta_{+-}$ would not depend on the placement of the dot along the CNT (provided the size of the dot is constant), the disorder-induced valley mixing would change sharply when the quantum dot would reach the disorder sites.

Another implementation of this idea would be to use two STM tips, which would create the two tunneling barriers defining a quantum dot. When they are shifted along the nanotube and the distance between them is kept fixed, the area of the quantum dot encounters different realizations of disorder. The amplitude of the $K K^{\prime}$ anticrossing would therefore also vary. The variable part of this splitting could then be attributed to disorder, and the constant part could be attributed to the boundary effect.
[1] R. Saito, G. Dresselhaus, and M. S. Dresselhaus, Physical Properties of Carbon Nanotubes (Imperial College Press, London, 1998).

[2] Understanding Carbon Nanotubes: From Basics to Applications, Lecture Notes in Physics, edited by A. Loiseau, P. Launois-Bernede, P. Petit, S. Roche, and J.-P. Salvetat (Springer, New York, 2006).

[3] J. Cuevas and E. Scheer, Molecular Electronics: An Introduction to Theory and Experiment, World Scientific Series in Nanotechnology and Nanoscience (World Scientific, Singapore, 2010).

[4] A. Makarovski, L. An, J. Liu, and G. Finkelstein, Phys. Rev. B 74, 155431 (2006).

[5] F. Kuemmeth, S. Ilani, D. Ralph, and P. McEuen, Nature (London) 452, 448 (2008).

[6] F. Pei, E. Laird, G. Steele, and L. Kouwenhoven, Nat. Nanotechnol. 7, 630 (2012).

[7] E. A. Laird, F. Kuemmeth, G. A. Steele, K. Grove-Rasmussen, J. Nygård, K. Flensberg, and L. P. Kouwenhoven, Rev. Mod. Phys. 87, 703 (2015).

[8] A. Rubio, D. Sánchez-Portal, E. Artacho, P. Ordejón, and J. M. Soler, Phys. Rev. Lett. 82, 3520 (1999).

[9] J. Jiang, J. Dong, and D.Y. Xing, Phys. Rev. B 65, 245418 (2002).

[10] L. Mayrhofer and M. Grifoni, Europhys. J. B 63, 43 (2008).

[11] K. Sasaki, S. Murakami, R. Saito, and Y. Kawazoe, Phys. Rev. B 71, 195401 (2005).

[12] M. Marganska, M. del Valle, S.H. Jhang, C. Strunk, and M. Grifoni, Phys. Rev. B 83, 193407 (2011).

[13] S. Bachilo, M. Strano, C. Kittrell, R. Hauge, R. Smalley, and R. Weisman, Science 298, 2361 (2002).

[14] A. M. Lunde, K. Flensberg, and A.-P. Jauho, Phys. Rev. B 71, 125408 (2005).
[15] E. McCann and V. Fal'ko, J. Phys.: Condens. Matter 16, 2371 (2004).

[16] W. Izumida, A. Vikström, and R. Saito, Phys. Rev. B 85, 165430 (2012).

[17] A. R. Akhmerov and C. W. J. Beenakker, Phys. Rev. B 77, 085423 (2008).

[18] T. Jespersen, K. Grove-Rasmussen, J. Paaske, K. Muraki, T. Fujisawa, J. Nygård, and K. Flensberg, Nat. Phys. 7, 348 (2011).

[19] K. Grove-Rasmussen, S. Grap, J. Paaske, K. Flensberg, S. Andergassen, V. Meden, H.I. Jørgensen, K. Muraki, and T. Fujisawa, Phys. Rev. Lett. 108, 176802 (2012).

[20] D. R. Schmid, S. Smirnov, M. Margańska, A. Dirnaichner, P. L. Stiller, M. Grifoni, A. K. Hüttel, and C. Strunk, Phys. Rev. B 91, 155435 (2015).

[21] T. Ando, J. Phys. Soc. Jpn 69, 1757 (2000).

[22] M. del Valle, M. Marganska, and M. Grifoni, Phys. Rev. B 84, 165427 (2011).

[23] C. T. White, D. H. Robertson, and J. W. Mintmire, Phys. Rev. B 47, 5485 (1993).

[24] M. Damnjanović, I. Milošević, T. Vuković, and R. Sredanović, Phys. Rev. B 60, 2728 (1999).

[25] E. B. Barros, A. Jorio, G. G. Samsonidze, R. B. Capaz, A. G. S. Filho, J. M. Filho, G. Dresselhaus, and M. S. Dresselhaus, Phys. Rep. 431, 261 (2006).

[26] D. Tománek and S. G. Louie, Phys. Rev. B 37, 8327 (1988).

[27] A. H. Castro Neto, F. Guinea, N. M. R. Peres, K. S. Novoselov, and A. Geim, Rev. Mod. Phys. 81, 109 (2009).

[28] G. Cuniberti, F. Grossmann, and R. Gutiérrez, Adv. Solid State Phys. 42, 133 (2002).

[29] D. S. Fisher and P. A. Lee, Phys. Rev. B 23, 6851 (1981). 
[30] The dimensionless parameter $\delta$ measuring the spin-orbit coupling strength [21,22] was taken to be $\delta=-2 \times 10^{-4}$.

[31] E. Laird, F. Pei, and L. Kouwenhoven, Nat. Nanotechnol. 8, 565 (2013).

[32] J.D. Sau and S. Tewari, Phys. Rev. B 88, 054503 (2013).

[33] W. Izumida, R. Okuyama, and R. Saito, Phys. Rev. B 91, 235442 (2015).

[34] A. Pályi and G. Burkard, Phys. Rev. Lett. 106, 086801 (2011).
[35] P. G. Collins, in Oxford Handbook of Nanoscience and Technology, Vol. 2 of Materials: Structures, Properties and Characterization Techniques, edited by A. Narlikar and Y. Fu (Oxford University Press, Oxford, 2010).

[36] A. Benyamini, A. Hamo, S. V. Kusminskiy, F. von Oppen, and S. Ilani, Nat. Phys. 10, 151 (2014).

[37] B. J. LeRoy, S. G. Lemay, J. Kong, and C. Dekker, Nature (London) 432, 371 (2004). 